\title{
Complete Genome Sequence of Canine Papillomavirus Type 9
}

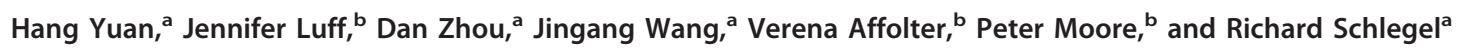 \\ Department of Pathology, Georgetown University Medical School, Washington, DC, USA, a and Department of Pathology, Microbiology and Immunology, School of \\ Veterinary Medicine, University of California, Davis, California, USA ${ }^{\mathrm{b}}$
}

\begin{abstract}
Papillomaviruses are nonenveloped, double-stranded DNA viruses that are associated with both benign and malignant tumors in animals and humans. We report the complete genome sequence of canine papillomavirus type 9 isolated from a solitary pigmented plaque on a mixed-breed bloodhound.
\end{abstract}

Pant apillomaviruses (PVs) are nonenveloped, double-stranded DNA viruses that are associated with both benign and malignant tumors in humans. For example, while the low-risk human papillomaviruses (HPVs) are known to cause benign tumors, such as warts (verrucae), on epidermal and mucosal surfaces, the high-risk HPVs induce lesions that can progress to cancers of the cervix, vulva, vagina, penis, anus, and oropharynx. In addition, HPVs are associated with specific epidermal cancers (11).

Papillomavirus infections are widespread in nature and occur in most mammalian species as well as in birds and turtles (1). These viral infections are highly host and tissue tropic and are rarely transmitted between species. The existence of relevant mammalian models for PV infections has made possible studies of PV evolution and pathogenesis and its life cycle. The canine model is one of the best systems for studying both epidermal and mucosal PV infections, and the analysis of canine oral papillomavirus (CPV-1) was critical to understanding host immunity against papillomavirus infection $(3,5)$, HPV vaccine development $(7,9)$, and viral oncogenesis $(2,4,8,10)$. To date, there are eight canine PVs whose genomes have been sequenced (6), some of which are associated with subclinical infections that can be become clinically manifest during immunosuppression. PV infections in immunosuppressed dogs have been noted to progress to aggressive squamous cell carcinomas.

Here we report the complete genome sequencing of a novel type of canine papillomavirus, designated canine papillomavirus type 9 (CPV-9), that was isolated from a solitary pigmented plaque on the left hind leg of a mixed-breed bloodhound. The viral DNA was isolated from this tumor by routine methods. Initially, PCR with general primers was used to amplify potential PV genome fragments. Sequencing of the products revealed an unknown CPV. To further pursue the type of CPV present, we used rolling circle amplification to generate the complete viral genome. The genome was then cloned into a pUC19 vector, and primer walking was used to sequence the entire genome from both directions. The sequence was analyzed using ABI 3730xl DNA-analyzing instruments for capillary electrophoresis and fluorescent dye terminator detection. Sequence contigs containing high-quality trace files were assembled using Vector NTI Advance 10 software (Invitrogen). Sequencing confirmed the presence of a novel CPV.

The complete genome sequence of CPV-9 is 7,873 bp. Similar to other papillomaviruses, CPV-9 has all of its open reading frames (ORFs) on the same coding strand of its circular double-stranded DNA genome. CPV-9 has seven ORFs that encode five early (E) proteins: E1, E2, E4, E6, and E7. In addition, there are two late (L) proteins: L1 and L2. The L1 gene is the most conserved gene within the papillomavirus genome and has therefore been used for the identifi- cation of new PV types. A new PV isolate is recognized if the DNA sequence of the L1 ORF differs by more than $10 \%$ from the closest known PV type (1). The L1 DNA of CPV-9 is most closely related (72\% homology) to the recently discovered CPV-4. These data will facilitate future investigations of the evolutionary characteristics and molecular pathogenesis of CPVs.

Nucleotide sequence accession number. The complete genome sequence of canine papillomavirus type 9 (CPV-9) is available in GenBank under accession number NC_016074.1.

\section{ACKNOWLEDGMENTS}

This project was supported by the National Center for Research Resources and the Office of Research Infrastructure Programs (ORIP) of the National Institutes of Health through grant 1R01RR032315-01.

\section{REFERENCES}

1. Bernard HU, et al. 2010. Classification of papillomaviruses (PVs) based on 189 PV types and proposal of taxonomic amendments. Virology 401:70-79.

2. Condjella R, et al. 2009. The canine papillomavirus e5 protein signals from the endoplasmic reticulum. J. Virol. 83:12833-12841.

3. Ghim S, et al. 2000. Spontaneously regressing oral papillomas induce systemic antibodies that neutralize canine oral papillomavirus. Exp. Mol. Pathol. 68:147-151.

4. Goldschmidt MH, et al. 2006. Severe papillomavirus infection progressing to metastatic squamous cell carcinoma in bone marrow-transplanted X-linked SCID dogs. J. Virol. 80:6621-6628.

5. Jain S, Moore RA, Anderson DM, Gough GW, Stanley MA. 2006. Cellmediated immune responses to COPV early proteins. Virology 356:23-34.

6. Lange CE, Tobler K, Lehner A, Vetsch E, Favrot C. 2012. A case of a canine pigmented plaque associated with the presence of a Chipapillomavirus. Vet. Dermatol. 23:76-80.

7. Suzich JA, et al. 1995. Systemic immunization with papillomavirus L1 protein completely prevents the development of viral mucosal papillomas. Proc. Natl. Acad. Sci. U. S. A. 92:11553-11557.

8. Wang J, Zhou D, Prabhu A, Schlegel R, Yuan H. 2010. The canine papillomavirus and gamma HPV E7 proteins use an alternative domain to bind and destabilize the retinoblastoma protein. PLoS Pathog. 6:e1001089.

9. Yuan H, et al. 2001. Immunization with a pentameric L1 fusion protein protects against papillomavirus infection. J. Virol. 75:7848-7853.

10. Yuan H, et al. 2007. An epidermotropic canine papillomavirus with malignant potential contains an E5 gene and establishes a unique genus. Virology 359:28-36.

11. zur Hausen H. 2009. Papillomaviruses in the causation of human cancers—a brief historical account. Virology 384:260-265.

Received 1 March 2012 Accepted 5 March 2012

Address correspondence to Hang Yuan, yuanh@georgetown.edu.

Copyright $\odot$ 2012, American Society for Microbiology. All Rights Reserved.

doi:10.1128/JVI.00543-12 